\title{
Calcul de l'irradiation externe dans une mine d'uranium
}

\author{
N. FOURCADE-CANCELLE (*)
}

(Manuscrit reçu le 5 mars 1975)

\begin{abstract}
RÉSUMÉ
Le débit de dose mesuré au centre d'une galerie creusée dans un minerai à $1 \mathrm{p}$. mille d'uranium était estimé, en moyenne, à $0,5 \mathrm{mrad} / \mathrm{h}$. On se propose ici de vérifier cette valeur par le calcul en tenant compte de la géométrie des sources et des principaux émetteurs de la famille de l'uranium.

On a calculé le débit de dose par irradiation externe dans deux cas types : - dans une galerie cylindrique de $50 \mathrm{~m}$ de long et de $2 \mathrm{~m}$ de diamètre; - dans une carrière à ciel ouvert de $50 \mathrm{~m}$ de long constituée par une paroi verticale de $2 \mathrm{~m}$ de haut et par une plate-forme de $8 \mathrm{~m}$ de large.

On a ainsi calculé les débits de dose à proximité des parois en tenant compte des différents rayonnements $\gamma$ et $\beta$ émis soit par les parois, soit par le radon diffusé dans l'atmosphère, soit par la poussière de minerai déposée sur la peau. Les valeurs obtenues par le calcul sont proches des valeurs mesurées. La contribution des rayonnements dépend du mode d'irradiation; ainsi, devant les parois de grande épaisseur, l'irradiation est due principalement aux rayonnements $\gamma$, tandis qu'en atmosphère contaminée de radon ou dans le cas de contamination cutanée, les rayonnements $\beta$ prédominent.
\end{abstract}

\section{SUMMARY}

The dose rate measured at the centre of a tunnel excavated in an ore at $1 \mathrm{p}$. thousand of uranium was assessed to average $0.5 \mathrm{mrad} / \mathrm{h}$. This value was verified by calculations taking account of the source geometries and the main emitters of the uranium family. The dose rate from external exposure was calculated for two typical cases:-in a cylindrical tunnel (length $=50 \mathrm{~m}$,

(*) Commissariat à l'Énergie atomique, Département de Protection, Service technique d'Études de Protection et de Pollution atmosphérique, B.P. n 6, 92260 Fontenay-aux-Roses.

RADIOPROTECTION, VOL. $10-\mathrm{N}^{\circ} 1$ 
diameter: $2 \mathrm{~m}$ ); -in an open quarry $50 \mathrm{~m}$ long, with a vertical wall, $2 \mathrm{~m}$ high, and a platform. $8 \mathrm{~m}$ wide. The dose rates were calculated close to the walls, taking account of the various $\gamma$ and $\beta$ radiation emitted either by the walls or by atmospheric radon or by the ore dust deposited on the skin. The calculated values were close to the measured values. The radiation contribution depends on the conditions of exposure; exposure is mainly due to $\gamma$ radiation close to very thick walls whereas $\beta$ radiation is prevailing in a contaminated atmosphere or in the case of skin contamination.

Pour estimer l'irradiation externe à laquelle on peut s'attendre dans une mine d'uranium on utilise couramment une relation simple tirée de mesures faites sur place il y a déjà de nombreuses années : au centre d'une galerie creusée dans un minerai à $1 \mathrm{p}$. mille le débit de dose est de $0,5 \mathrm{mrard} / \mathrm{h}[1]$.

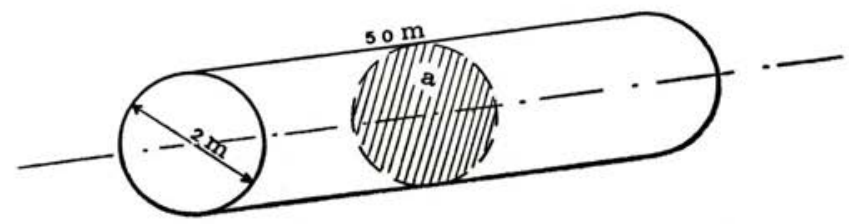

FIG. 1. - Schéma de la galerie.

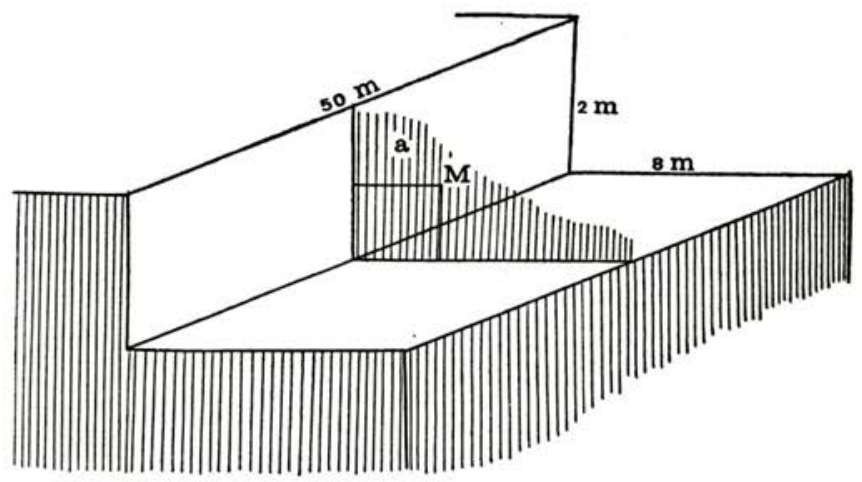

Fig. 2. - Schéma de la carrière.

(a) Les débits de`đose ont été calculés dans le plan de symétrie.

Les conditions de telles》mesures étant souvent incertaines, on s'est proposé ici de vérifier cette règle empirique en calculant le débit de dose reçu des parois dans deux cas types, assez représentatifs de cas réels ( fig. 1 et 2) :

- dans une galerie cylindrique de $50 \mathrm{~m}$ de long et de $2 \mathrm{~m}$ de diamètre creusée dans un minerai de grande épaisseur et à teneur uniforme de $1 \mathrm{p}$. mille; 
- dans une carrière à ciel ouvert, de $50 \mathrm{~m}$ de long, constituée par une paroi verticale de $2 \mathrm{~m}$ de haut et par une plate-forme horizontale de $8 \mathrm{~m}$ de large.

En même temps on a calculé :

- l'irradiation externe résultant d'une concentration atmosphérique de $1.10^{-7} \mathrm{Ci} / \mathrm{m}^{3}$ en radon;

- l'irradiation produite par une contamination cutanée de $10^{-6} \mu \mathrm{Ci} / \mathrm{cm}^{2}$ en poussières de minerai.

\section{PRINCIPAUX RÉSULTATS}

TABLEAU I

DÉbIT DE DOSE PROduIt PAR DU Minerai D'URANIUM A 1 P. MILle.

\begin{tabular}{|c|c|c|c|}
\hline Débit de dose en mrad/h & $\gamma$ & $\beta$ & Total \\
\hline $\begin{array}{l}\text { reçu des parois par les tissus profonds (a) } \\
\text { au centre de la galerie }\end{array}$ & 0,65 & 0,12 & 0,77 \\
\hline $\begin{array}{l}\text { reçu des parois par les tissus profonds, } \\
\text { dans la carrière, à } 1 \text { mètre du mur } \\
\text { et du sol }\end{array}$ & 0,5 & 0,12 & 0,62 \\
\hline $\begin{array}{l}\text { reçu par les tissus profonds, d'une } \\
\text { concentration atmosphérique en radon } \\
\text { égale à } 1 \mathrm{CMA}\left(10^{-7} \mathrm{Ci} / \mathrm{m}^{3}\right)\end{array}$ & $1,2 \cdot 10^{-3}$ & $1,5 \cdot 10^{-2}$ & $1,6 \cdot 10^{-2}$ \\
\hline $\begin{array}{l}\text { reçu par la peau, d'une contamination } \\
\text { cutanée de } 10^{-6} \mu \mathrm{Ci} / \mathrm{cm}^{2} \text { en poussières } \\
\text { de minerai }\end{array}$ & $6,8 \cdot 10^{-5}$ & $6,5 \cdot 10^{-3}$ & $6,6 \cdot 10^{-3}$ \\
\hline
\end{tabular}

(a) c'est-à-dire sous $300 \mathrm{mg} / \mathrm{cm}^{2}$

Dans ce tableau, le débit de dose reçu des parois de la galerie ou de la carrière est celui qui atteindrait un dosimètre porté sur le vêtement. Son calcul tient compte de l'atténuation par le corps d'une partie des rayonnements émis. L'expérience montre que les valeurs de débit de dose calculées sont presque toujours un peu supérieures (d'environ $20 \mathrm{p}$. cent) aux valeurs mesurées. La règle rappelée au début de cette note donne donc de façon particulièrement simple et commode une bonne approximation. Les tableaux II et III donnent les valeurs du débit de dose $\gamma$ reçu des parois par l'organisme entier en d'autres emplacements dans la section médiane ou dans la carrière :

voL. $10-\mathrm{N}^{\circ} 1$ 
TABLEAU II

DÉBIT DE DOSE A DIFFÉRENTES HAUTEURS DANS LA GALERIE

\begin{tabular}{c|c}
\hline \hline $\begin{array}{c}\text { Hauteur au-dessus du sol } \\
\text { (en cm) }\end{array}$ & $\begin{array}{c}\text { Débit de dose } \gamma \\
\text { (en mrad/h) }\end{array}$ \\
\hline $100(=\mathrm{R})$ & 0,65 \\
70 & 0,65 \\
50 & 0,69 \\
30 & 0,76 \\
10 & 0,92 \\
5 & 1,08 \\
\hline \hline
\end{tabular}

TABLEAU III

DÉBIT DE DOSE EN DIFFÉRENTS ENDROITS DANS LA CARRIÈRE.

\begin{tabular}{l|c|c}
\hline \hline & à $1 \mathrm{~m} \mathrm{du} \mathrm{mur}$ & à $3 \mathrm{~m} \mathrm{du} \cdot \mathrm{mur}$ \\
\hline à $150 \mathrm{~cm}$ du sol & $0,46 \mathrm{mrad} / \mathrm{h}$ & $0,46 \mathrm{mrad} / \mathrm{h}$ \\
à $100 \mathrm{~cm}$ du sol & $0,50 \mathrm{mrad} / \mathrm{h}$ & $0,47 \mathrm{mrad} / \mathrm{h}$ \\
à $50 \mathrm{~cm}$ du sol & $0,65 \mathrm{mrad} / \mathrm{h}$ & $0,63 \mathrm{mrad} / \mathrm{h}$ \\
\hline \hline
\end{tabular}

\section{COMMENTAIRES}

$1^{\circ}$ On trouvera dans les annexes A, B et $\mathrm{C}$ les méthodes et les éléments utilisés pour le calcul de ces résultats.

$2^{\circ} \mathrm{La}$ contribution du rayonnement $\beta$ à la dose reçue des parois par les tissus profonds est assez faible : 15 à $19 \mathrm{p}$. cent de la dose totale, à $1 \mathrm{~m}$ de la paroi. Ce résultat est en bon accord avec les mesures faites sur un bloc de minerai (voir annexe C). La part du rayonnement $\beta$ augmente légèrement - jusqu'à 20 p. cent - quand on se rapproche d'une paroi.

$3^{\circ}$ Par contre, la dose délivrée sous la peau par le radon atmosphérique (supposé en équilibre avec ses descendants) est due pour les $9 / 10^{\mathrm{e}}$ au rayonnement $\beta$. Mais cette dose, pour 1 CMA de radon, est plus de dix fois inférieure à la dose provenant des parois.

$4^{\circ}$ Nous avons supposé que la galerie avait $2 \mathrm{~m}$ de diamètre et $50 \mathrm{~m}$ de long. Mais on peut voir dans l'annexe A que les résultats sont pratiquement indépendants du diamètre de la galerie et qu'ils ne changeraient pas si sa longueur devenait infinie.

$5^{\circ}$ La densité du minerai supposée pour nos calculs est de 2,6. Si cette densité augmentait sans que la composition massique change, le débit de dose resterait le même car l'intensité de source surfacique $S_{A}$ est égale à $S_{v} / \mu_{s}$ (voir 


\section{'punelec}

2. PETITE PLACE - 78000 VERSAILLES

Tél. 951-29-30

CHAMBRE A GRILLE POUR SPECTROMETRIE ALPHA
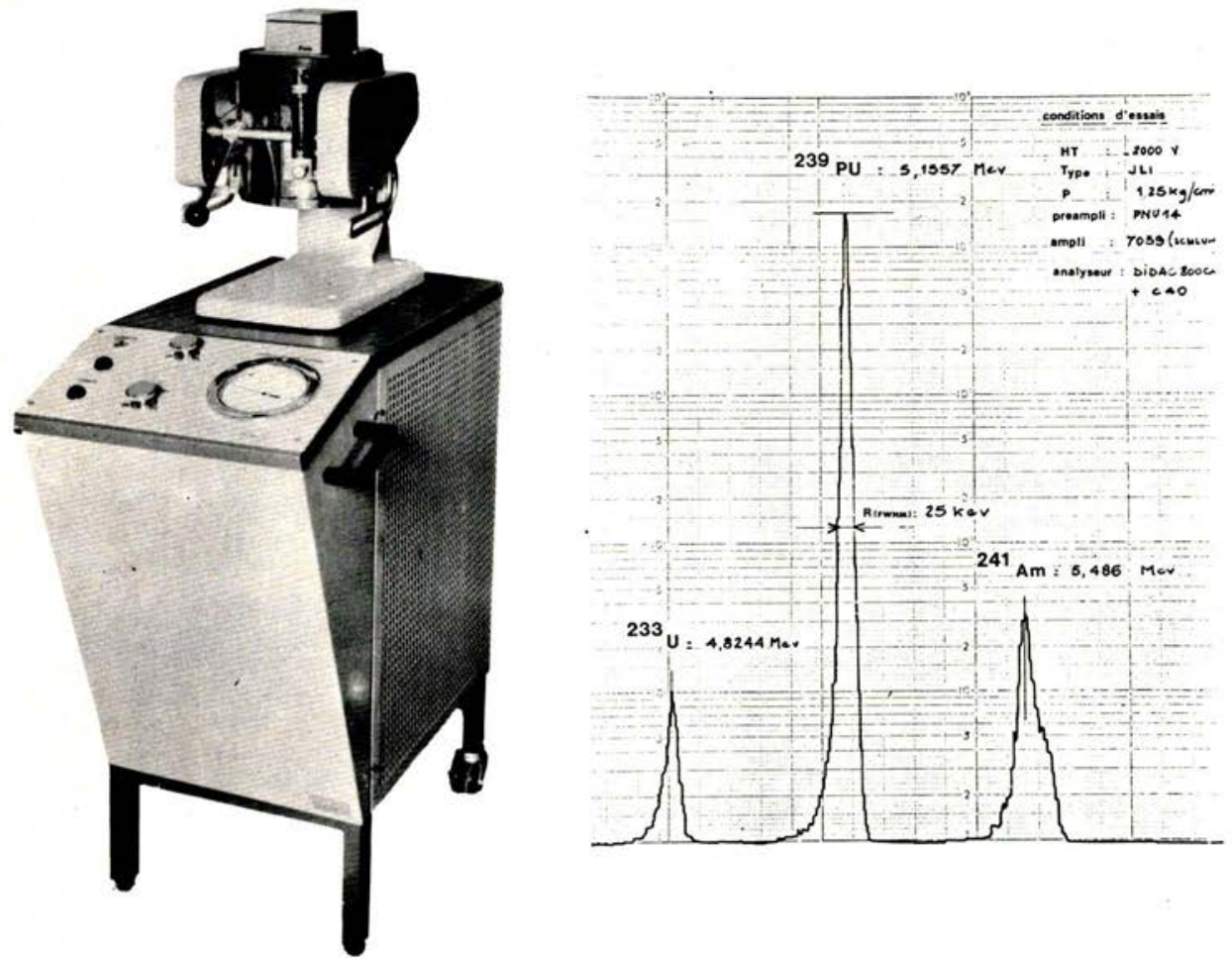

- RESOLUTION GARANTIE: $25 \mathrm{KeV}$ SUR $5 \mathrm{MeV}$

. SURFACE: $5000 \mathrm{~mm}^{2}$

- LIMITE DE DETECTION : 10 FEMTOCURIES DE PLUTONIUM $239\left(10.10^{-15} \mathrm{CURIES}^{-10}\right.$ 


\section{SPECIFICATIONS}

\section{Chambre}

Géométrie $2 \pi$

Diamètre maximal : $80 \mathrm{~mm}$ (surface $5000 \mathrm{~mm}^{2}$ )

Temps de collection : $0,5 \mu \mathrm{s}$

Bruit de fond $\quad: 2$ coups par heure 4 et $6 \mathrm{MeV}$

Gaz argon méthane (à $10 \%$ de $\mathrm{CH}^{4}$ )

Bouteilies

: $10 \mathrm{~m}^{3}$

Pression

Débit après dégazage initial

: 0,5 à $1,2 \mathrm{~kg} / \mathrm{cm}^{2}$

: 6 litres/heure

Préamplificateur (PNU 14 inclus dans NU 14-B)

Transistor d'entrée à effet de champ : $>10^{\prime \prime} \Omega$

Bruit équivalent à l'entrée $\quad: 0,6 \mu \mathrm{V}$

Bruit à mi-hauteur $\quad:<17 \mathrm{KeV}$

Bruit en nombre d'électrons injectés à l'entrée $\sim 2000$ Impédance d'entrée

Sortie négative

: $50 \Omega$

Temps de montée

: $200 \mathrm{mV}$

Temps de descente

: $0,5 \mu \mathrm{s}$

Alimentations

: $30 \mu \mathrm{s}$

: $+24 \mathrm{~V}(42 \mathrm{~mA})$

$-24 \mathrm{~V}(27 \mathrm{~mA})$ 
annexe A) où $S_{v}$ (intensité de source volumique) et $\mu_{s}$ (coefficient macroscopique d'absorption $\gamma$ du minerai) sont tous deux proportionnels à la densité.

En réalité, lorsque la teneur du minerai augmente, la densité augmente mais la composition massique change avec l'enrichissement en uranium; le coefficient massique d'absorption $\gamma$ de l'uranium étant plus fort que celui de la silice, cet enrichissement conduit, en fait, à une augmentation de $\mu_{s}$ un peu plus rapide que celle de $S_{v}$, donc à une légère diminution de $S_{A}$. Par exemple, avec un minerai à $30 \mathrm{p}$. cent on a une densité de 3,5 et un débit de dose, pour une teneur ramenée à $1 \mathrm{p}$. mille, inférieur de $9 \mathrm{p}$. cent au débit de dose calculé avec le minerai de densité 2,6.

On commet donc une légère erreur par excès dans l'estimation du débit de dose rapporté à la teneur de $1 \mathrm{p}$. mille quand on admet que le débit de dose ne change pas lorsque la densité augmente. 


\section{ANNEXE A \\ CALCUL DE L'IRRADIATION EXTERNE \\ PAR LE RAYONNEMENT $\gamma$}


TABLEAU IV

Principaux Émetteurs $\gamma$ de LA famille de L'URANium.

\begin{tabular}{|c|c|c|c|}
\hline \multirow{2}{*}{ Emetteurs } & \multicolumn{2}{|c|}{ Photons émis [2] } & \multirow{2}{*}{$\begin{array}{l}\text { Nombre de photons } \\
\text { émis par seconde } \\
\text { pour } 1 \mathrm{~kg} \mathrm{~d} \text { 'U } \\
\text { nat. (a) }\end{array}$} \\
\hline & énergie en $\mathrm{MeV}$ & $\%$ & \\
\hline${ }^{238} \mathrm{U}$ & 0,048 & 23 & $2,82.10^{6}$ \\
\hline $234 \mathrm{Th}$ & $\begin{array}{l}0,09 \\
0,06 \\
0,03\end{array}$ & $\begin{array}{l}15 \\
6,5 \\
6,5\end{array}$ & $\begin{array}{r}1,85.10^{6} \\
8.10^{5} \\
8.10^{5}\end{array}$ \\
\hline $234 \mathrm{~m}_{\mathrm{Pa}}$ [3] & $\begin{array}{l}1,83 \\
1,01 \\
0,8 \\
0,77\end{array}$ & $\begin{array}{l}0,3 \\
1,5 \\
0,16 \\
0,87\end{array}$ & $\begin{array}{l}3,7 \cdot 10^{4} \\
1,85 \cdot 10^{5} \\
1,97 \cdot 10^{4} \\
1,07 \cdot 10^{5}\end{array}$ \\
\hline $\begin{array}{ll}226 & \mathrm{Ra} \\
222 & \mathrm{Rn} \\
214 & \mathrm{~Pb}\end{array}$ & $\begin{array}{l}0,186 \\
0,325 \\
0,295 \\
0,352\end{array}$ & $\begin{array}{r}4 \\
4 \\
19 \\
36\end{array}$ & $\begin{array}{l}4,9.10^{5} \\
4,9.10^{5} \\
2,34 \cdot 10^{6} \\
4,43 \cdot 10^{6}\end{array}$ \\
\hline $214 \mathrm{Bi}$ & $\begin{array}{l}0,609 \\
0,769 \\
0,935 \\
1,12 \\
1,238 \\
1,378 \\
1,4 \\
1,509 \\
1,728 \\
1,764 \\
1,848 \\
2,117 \\
2,204 \\
2,445\end{array}$ & $\begin{array}{r}47 \\
5 \\
3 \\
17 \\
6 \\
5 \\
4 \\
2 \\
3 \\
17 \\
2 \\
1 \\
5 \\
2\end{array}$ & $\begin{array}{ll}5,8 & .10^{6} \\
6,15 & \cdot 10^{5} \\
3,7 & \cdot 10^{5} \\
2,1 & .10^{6} \\
7,4 & \cdot 10^{5} \\
6,1 & .10^{5} \\
4,9 & .10^{5} \\
2,4 & .10^{5} \\
3,7 & .10^{5} \\
2,1 & .10^{6} \\
2,4 & .10^{5} \\
1,2 & .10^{5} \\
6,2 & .10^{5} \\
2,5 & .10^{5}\end{array}$ \\
\hline${ }^{235} \mathrm{U}$ & $\begin{array}{l}0,14 \\
0,185 \\
0,204\end{array}$ & $\begin{array}{r}4 \\
54 \\
5\end{array}$ & $\begin{array}{ll}2,3 & .10^{4} \\
3 & .10^{5} \\
2,8 & .10^{4}\end{array}$ \\
\hline${ }^{231} \mathrm{~Pa}$ & 0,29 & 6 & $3,4 \cdot 10^{4}$ \\
\hline${ }^{227} \mathrm{Th}$ & $\begin{array}{l}0,24 \\
0,3\end{array}$ & $\begin{array}{r}15 \\
8\end{array}$ & $\begin{array}{ll}8,5 & .10^{4} \\
4,5 & .10^{4}\end{array}$ \\
\hline${ }^{223} \mathrm{Ra}$ & $\begin{array}{l}0,15 \\
0,3\end{array}$ & $\begin{array}{l}10 \\
16\end{array}$ & $\begin{array}{ll}5,7 & .10^{4} \\
9 & .10^{4}\end{array}$ \\
\hline $211 \mathrm{~Pb}$ & $\begin{array}{l}0,4 \\
0,8\end{array}$ & $\begin{array}{l}5,2 \\
4,4\end{array}$ & $\begin{array}{ll}3 & .10^{4} \\
2,5 & .10^{4}\end{array}$ \\
\hline $211_{\mathrm{Bi}}$ & 0,35 & 14 & $7,9.10^{4}$ \\
\hline${ }^{211} \mathrm{Po}$ & $\begin{array}{l}0,57 \\
0,9 \\
\end{array}$ & $\begin{array}{l}0,5 \\
0,5 \\
\end{array}$ & $\begin{array}{ll}2,8 & .10^{3} \\
2,8 & .10^{3} \\
\end{array}$ \\
\hline
\end{tabular}

(a) on suppose que l'uranium est en équilibre radioactif avec ses produits de filiation et que tous ceux-ci, y compris le radon et ses descendants, se trouvent dans le minerai. 
Pour la commodité du calcul on peut, sans commettre d'erreur par défaut, réduire cet ensemble à cinq groupes d'énergie :

TABLEAU $V$

ÉMISSIONS, PAR GROUPES D'ÉNERGIE.

\begin{tabular}{c|c|c}
\hline \hline \multirow{2}{*}{$\begin{array}{c}\text { Energie moyenne } \\
\text { MeV }\end{array}$} & \multicolumn{2}{|c}{ Nombre de photons émis par seconde } \\
\cline { 2 - 3 } & par kg d'U nat. & $\begin{array}{c}\text { par cm } \mathrm{cm}^{3} \text { de minerai } \\
\text { (densité }: 2,6 \\
\text { teneur }: 1 \text { p. mille) }\end{array}$ \\
\hline 0,15 & $2,5.10^{6} \gamma \cdot \mathrm{s}^{-1}$ & $\mathrm{~S}_{\mathrm{v}}=6,5 \gamma \cdot \mathrm{s}^{-1} \mathrm{~cm}^{-3}$ \\
0,3 & $7,6.10^{6}$ & $1,93.10^{1}$ \\
0,6 & $6,4.10^{6}$ & $1,66.10^{1}$ \\
1,1 & $4,1.10^{6}$ & $1,07 \cdot 10^{1}$ \\
1,8 & $4,5.10^{6}$ & $1,17.10^{1}$ \\
\hline \hline
\end{tabular}

\section{IRRADIATION PAR LES PAROIS}

\section{Mode DE CALCUL}

La masse de minerai dans laquelle est creusée la galerie ou la carrière est une source de grande épaisseur, limitée par une paroi, d'intensité volumique $S_{v}$ et de coefficient d'absorption $\mu_{s}$. Elle est équivalente à une source superficielle d'intensité surfacique $S_{A}=S_{v} / \mu_{s}[9$, p. 305].

La paroi cylindrique de la galerie est formée de $2 \pi R$ bandes parallèles à son axe, de $50 \mathrm{~m}$ de long et de $1 \mathrm{~cm}$ de large. Chacune de ces bandes constitue une source linéaire d'intensité linéique $S_{L}=S_{A} \times 1 \mathrm{~cm}$, ce qui produit à une distance $a$ de son milieu un flux :

$$
\varphi=\frac{S_{L}}{2 \pi a} \theta \quad[3, \text { p. 367] }
$$

où $\theta$ est l'angle de tangente $L / 2 a$. Au centre de la galerie $(a=R)$ le flux provenant de toute la paroi, c'est-à-dire, des $2 \pi R$ bandes, est alors :

$$
\varphi=S_{L} \theta \text {. }
$$

De même, dans la carrière, le mur et la plate-forme sont décomposés en $n$ bandes étroites, de $50 \mathrm{~m}$ de long et de largeur $d$, chacune constituant une source linéaire d'intensité linéique $S_{L}=S_{A} \times d$ et distante de $a$ du point $M$ considéré. Il en résulte en $M$ un flux total :

$$
\varphi=\sum_{1}^{n} \frac{S_{L}}{2 \pi a_{i}} \theta_{i} .
$$


En toute rigueur, il faudrait tenir compte de l'absorption par l'air, ce qui donnerait pour expression du flux :

$$
\varphi=B \frac{S_{L}}{2 \pi a} F(\theta, b) \quad[4, \text { p. 348], }
$$

où $b$ est le produit $a \times \mu, \mu$ étant le coefficient d'absorption du milieu d'épaisseur $a$ (ici, ce milieu est l'air) et $B$ le ( build up ).

Mais comme $\mu$ est faible, l'absorption par l'air est négligeable aux petites distances $a$. Ainsi, pour $a$ inférieur ou égal à $3 \mathrm{~m}$, les expressions (1) et (4) donnent le même résultat; pour une distance $a$ de $5 \mathrm{~m}$, l'expression (1) surestime le débit de dose de $10 \mathrm{p}$. cent et de $15 \mathrm{p}$. cent si $a$ égale $10 \mathrm{~m}$.

On le vérifie dans le tableau VII où sont données les valeurs de $\theta$ et de $F$ $(\theta, b)$ en fonction de $a$.

Avec un ensemble de sources élémentaires - cas de l'expression (3) où $a_{i}$ varie de 1 à $7 \mathrm{~m}$, le débit de dose dû aux sources $i$ dont les distances $a_{i}$ sont supérieures à $4 \mathrm{~m}$ représente seulement $10 \mathrm{p}$. cent du débit de dose total; l'utilisation de l'expression simple (3) n'entraîne donc qu'une très faible erreur (de l'ordre de quelques p. cent).

La dose que nous considérons est celle qu'indiquera un dosimètre porté sur la poitrine. Dans la galerie, ce dosimètre voit directement une moitié de la source et voit l'autre moitié à travers le corps, qu'on peut assimiler à un écran de $20 \mathrm{~cm}$ d'eau. L'expression (2) devient alors :

$$
\varphi=S_{L} \theta\left(\frac{1}{2}+\frac{1}{2} B e^{-b}\right)
$$

$B e^{-b}$ est le facteur d'atténuation par l'écran d'eau; sa valeur est très voisine de 0,7 pour les cinq groupes d'énergie retenus.

Dans la carrière, seule une partie du sol est masquée par le corps. $\mathrm{Si}$ on voit directement $x$ sources lignes élémentaires, l'expression (3) devient :

$$
\varphi=\sum_{1}^{x} \frac{S_{L}}{2 \pi a_{i}} \theta_{i}+\sum_{x+1}^{n} \frac{S_{L}}{2 \pi a_{i}} \theta_{i} B e^{-b}
$$

C'est, en définitive, à l'aide de ces expressions (5) et (6) que nous calculons le débit de dose donné par les parois. On a, dans les tableaux VI et VII les principaux éléments nécessaires au calcul. L'intensité surfacique $S_{a}$ a été traduite en débit de dose, pour montrer la part de chaque groupe d'énergie dans la dose totale. 


\section{TABLEAU VI}

INTENSITÉ VOLUMIQUE ET SURFACIQUE.

\begin{tabular}{c|c|c|c|c|c}
\hline \hline $\begin{array}{c}\text { Energie } \\
\text { moyenne } \\
\mathrm{MeV}\end{array}$ & $\begin{array}{c}\gamma \cdot \mathrm{s}^{-1} \mathrm{~cm}_{\mathrm{V}} \\
\text { (voir tabl. V) }\end{array}$ & $\begin{array}{c}\mu_{\mathrm{s}} \\
\mathrm{cm}^{-1}\end{array}$ & $\begin{array}{c}\mathrm{S}_{\mathrm{A}} \\
=\mathrm{S}_{\mathrm{v}} / \mu_{\mathrm{S}} \\
\gamma \cdot \mathrm{s}^{-1} \mathrm{~cm}^{-2}\end{array}$ & $\begin{array}{c}\mathrm{rad} / \mathrm{h} \\
\text { pour } \\
1 \gamma \cdot \mathrm{s}^{-1} \mathrm{~cm}^{-2}\end{array}$ & $\begin{array}{c}\mathrm{S}_{\mathrm{A}} \\
\text { en rad/h }\end{array}$ \\
\hline 0,15 & 6,5 & 0,77 & 8,44 & $2,6 \cdot 10^{-7}$ & $2,2 \cdot 10^{-6}$ \\
0,3 & 19,7 & 0,30 & 65,6 & $5,4 \cdot 10^{-7}$ & $3,7 \cdot 10^{-5}$ \\
0,6 & 16,6 & 0,21 & 79 & $1,1.10^{-6}$ & $8,7 \cdot 10^{-5}$ \\
1,1 & 10,7 & 0,16 & 67 & $1,85 \cdot 10^{-6}$ & $1,24 \cdot 10^{-4}$ \\
1,8 & 11,7 & 0,12 & 97,5 & $2,7 \cdot 10^{-6}$ & $2,63 \cdot 10^{-4}$ \\
\hline \hline
\end{tabular}

TABLEAU VII

VAleurs de $b$ et $F(\theta, b)$ EN FONCtion de LA Distance a la source.

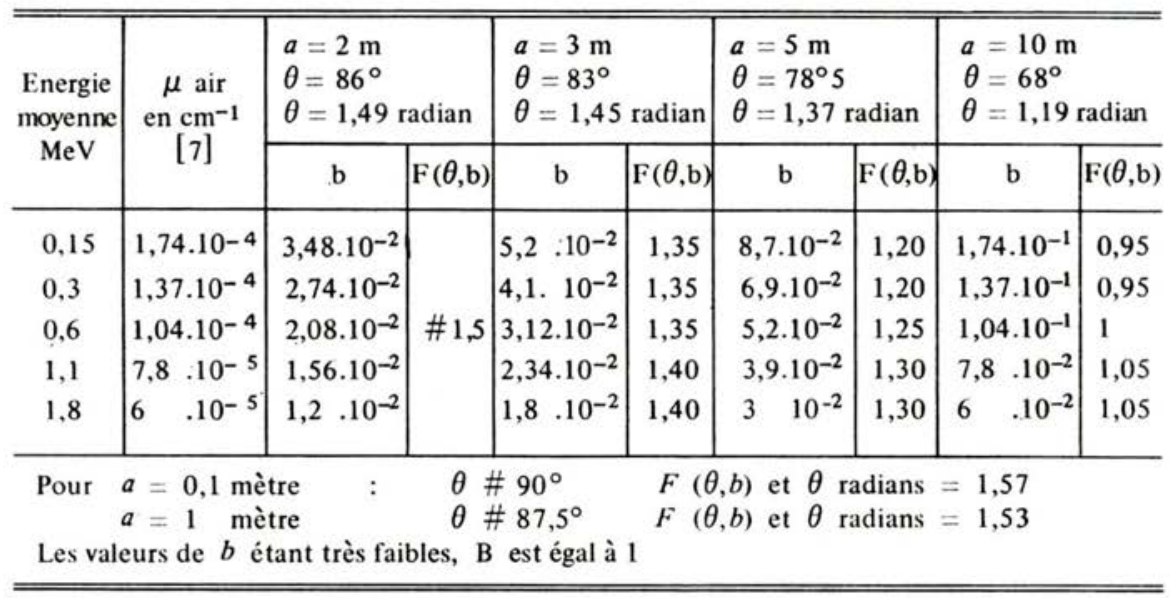

\section{REMARQUES}

$1^{\circ} \theta$ et $F(\theta, b)$ varient très peu lorsque $a$ varie de 0,1 à quelques mètres; en se reportant aux expressions (2) et (4) on voit alors que le débit de dose au centre de la galerie est, dans ces limites, pratiquement indépendant de la valeur du rayon.

$2^{\circ}$ Nous avons supposé que galerie et carrière étaient longues de $50 \mathrm{~m}$. $\mathrm{Si}$ cette longueur devenait infinie, la très faible variation de $\theta$ radians ou de $F(\theta, b)$ lorsque $\theta$ approche $90^{\circ}$ fait que le débit de dose à de faibles distances des parois (inférieures à quelques mètres) n'augmenterait pratiquement pas. 


\section{RÉSULTATS}

Le débit de dose $\gamma$ donné par les parois a été calculé en différents points de la section médiane de la galerie et de la carrière.

TABLEAU VIII

IRRADIATION EXTERNE DANS LA GALERIE.

\begin{tabular}{c|c}
\hline \hline $\begin{array}{c}\text { Hauteur au-dessus } \\
\text { du sol, en cm }\end{array}$ & $\begin{array}{c}\text { Débit de dose } \gamma \\
\text { en mrad/h }\end{array}$ \\
\hline 100 & 0,65 \\
70 & 0,65 \\
50 & 0,69 \\
30 & 0,76 \\
10 & 0,92 \\
5 & 1,08 \\
\hline
\end{tabular}

TABLEAU IX

IRRADIATION EXTERNE DANS LA CARRIÈRE.

\begin{tabular}{l|l|l}
\hline \hline & à $1 \mathrm{~m} \mathrm{du} \mathrm{muŕ}$ & à $3 \mathrm{~m}$ du mur \\
\hline à $150 \mathrm{~cm}$ du sol & $0,46 \mathrm{mrad} / \mathrm{h}$ & $0,46 \mathrm{mrad} / \mathrm{h}$ \\
à $100 \mathrm{~cm}$ du sol & $0,50 \mathrm{mrad} / \mathrm{h}$ & $0,47 \mathrm{mrad} / \mathrm{h}$ \\
à $50 \mathrm{~cm}$ du sol & $0,65 \mathrm{mrad} / \mathrm{h}$ & $0,63 \mathrm{mrad} / \mathrm{h}$ \\
\hline \hline
\end{tabular}

\section{IRRADIATION EXTERNE PAR LE RADON}

Le calcul de l'irradiation en atmosphère contaminée par le radon 222 a été fait en supposant que cet élément et ses produits de filiation étaient en équilibre radioactif et répartis uniformément. Les principaux de ces radionucléides figurent dans le tableau $\mathrm{X}$.

A teneur de minerai égale, on peut s'attendre à ce que la concentration en radon soit plus forte et plus uniforme dans l'atmosphère d'une galerie que dans la carrière à ciel ouvert. Aussi, nous bornerons-nous au cas de la galerie et nous supposons que la concentration en radon est de 1 CMA, c'est-à-dire, $10^{-10} \mathrm{Ci} / 1$ ou $10^{-7} \mathrm{Ci} / \mathrm{m}^{3}$.

La source se présente comme une source en volume cylindrique, de rayon $R$ égal à $1 \mathrm{~m}$ et de longueur $2 h$ égale à $50 \mathrm{~m}$. Le débit de dose en son centre se calcule à l'aide de l'expression suivante [3, p. 382] :

$$
\varphi=\frac{S_{v}}{2} h\left[\log \left(1+\frac{R^{2}}{h^{2}}\right)+\frac{2 R}{h} \operatorname{arctang} \frac{h}{R}\right] .
$$

VOL. $10-\mathrm{N}^{\circ} 1$ 
TABLEAU $\mathrm{X}$

Principales Émissions du RADON eT DE SES DESCENDANTS.

\begin{tabular}{|c|c|c|c|c|c|}
\hline \multirow{3}{*}{ Elément } & \multicolumn{3}{|c|}{ Mode de désintégration } & \multirow{2}{*}{\multicolumn{2}{|c|}{ Emission $\gamma$}} \\
\hline & \multirow[t]{2}{*}{$\alpha$} & \multicolumn{2}{|c|}{$\beta$} & & \\
\hline & & $\mathrm{E}(\mathrm{MeV})$ & $\%$ & $\mathrm{E}(\mathrm{MeV})$ & $\%$ \\
\hline $\begin{array}{l}\text { radon } 222 \\
\text { polonium } 218 \\
\text { (radium A) }\end{array}$ & $\begin{array}{l}\alpha \\
\alpha\end{array}$ & & & 0,325 & 4 \\
\hline $\begin{array}{l}\text { plomb } 214 \\
\text { (radium B) }\end{array}$ & & $\begin{array}{l}0,65 \\
0,59\end{array}$ & $\begin{array}{l}44 \\
56\end{array}$ & $\begin{array}{l}0,242 \\
0,295 \\
0,352\end{array}$ & $\begin{array}{r}4 \\
19 \\
36\end{array}$ \\
\hline $\begin{array}{l}\text { bismuth } 214 \\
\text { (radium C) }\end{array}$ & & $\begin{array}{l}3,26 \\
1,88 \\
1,51 \\
1 \\
0,4\end{array}$ & $\begin{array}{r}19 \\
9 \\
40 \\
23 \\
9\end{array}$ & $\begin{array}{l}0,609 \\
0,769 \\
0,935 \\
1,120 \\
1,238 \\
1,378 \\
1,4 \\
1,509 \\
1,728 \\
1,764 \\
1,848 \\
2,117 \\
2,204 \\
2,445\end{array}$ & $\begin{array}{r}47 \\
5 \\
3 \\
17 \\
6 \\
5 \\
4 \\
2 \\
3 \\
17 \\
2 \\
1 \\
5 \\
2\end{array}$ \\
\hline $\begin{array}{l}\text { polonium } 214 \\
\text { (radium C') }\end{array}$ & $\alpha$ & & & & \\
\hline $\begin{array}{l}\text { bismuth } 210 \mathrm{~m} \\
\text { polonium } 210\end{array}$ & $\alpha$ & 1,17 & 100 & & \\
\hline
\end{tabular}

L'autoabsorption dans l'air est négligée, mais on tient compte de l'absorption par le corps du flux issu de la moitié du volume source.

Comme précédemment, on a rangé les émetteurs $\gamma$ en cinq groupes d'énergie et on a calculé pour chaque énergie, la valeur de $S_{v}$ exprimée directement en millirad par heure pour $10^{-7} \mathrm{Ci}$ de radon par mètre cube.

$\mathrm{Au}$ centre de la galerie, la valeur de $\varphi$ est alors de $1,4.10^{-3} \mathrm{mrad} / \mathrm{h}$; la moitié du flux $\varphi$ étant atténué par un facteur 0,7 , le débit de dose est finalement :

$$
\varphi\left(\frac{1}{2}+\frac{1}{2} \cdot 0,7\right)=1,2 \cdot 10^{-3} \mathrm{mrad} / \mathrm{h} .
$$




\section{IRRADIATION $\gamma$ PAR CONTAMINATION CUTANÉE}

On suppose que la composition de la poussière déposée sur la peau est la même que celle du minerai exploité (teneur de $1 \mathrm{p}$. mille en uranium) et que l'activité surfacique de cette contamination est de $10^{-6} \mu \mathrm{Ci} \alpha / \mathrm{cm}^{2}$ (c'est l'ordre de grandeur de ce que l'on regarde habituellement comme limite admissible pour une contamination de surface par des émetteurs $\alpha$ ).

Le minerai considéré ayant une activité massique de $1,67 \cdot 10^{-3} \mu \mathrm{Ci} / \mathrm{g}$, la masse correspondant à cette contamination est de $0,6 \mathrm{mg} / \mathrm{cm}^{2}$; c'est une charge surfacique relativement forte, peu susceptible d'être dépassée sur une grande surface de peau et pendant une longue durée.

Cette couche de poussière peut être assimilée à une plaque mince de grandes dimensions, qui donne le flux :

$$
\varphi=B \frac{S_{A}}{2} E_{1}\left(b_{1}\right) \quad[3, \text { p. 397] }
$$

Dans cette expression, $b_{1}=\mu x$, $\mu$ est le coefficient linéaire d'absorption des tissus traversés (que l'on peut assimiler à de l'eau) et $x$ la profondeur à laquelle on veut calculer le débit de dose. On admet que les rayonnements qui traversent $7 \cdot 10^{-3} \mathrm{~cm}$ (ou $7 \mathrm{mg} / \mathrm{cm}^{2}$ ) atteignent la couche basale de l'épiderme et que ceux qui traversent $0,3 \mathrm{~cm}$ (ou $300 \mathrm{mg} / \mathrm{cm}^{2}$ ) atteignent les tissus profonds. La valeur de $b_{1}$ étant très faible, $B$ est très peu différent de 1 .

Le calcul est résumé dans le tableau XI.

TABLEAU XI

Débit DE DOSE $\varphi$ REÇU PAR LA PEAU ET Les TISSUS PROFONDS.

\begin{tabular}{|c|c|c|c|c|c|c|}
\hline \multirow{2}{*}{$\begin{array}{c}\mathrm{E} \\
(\mathrm{MeV})\end{array}$} & \multirow{2}{*}{$\begin{array}{l}\text { Photons émis } \\
\text { par seconde } \\
\text { pour } 0,6 \mathrm{mg} \\
\text { de minerai } \\
\text { (cf. tableau V) }\end{array}$} & \multirow{2}{*}{$\underset{\mathrm{mrad} / \mathrm{h}}{\mathrm{S}_{\mathrm{A}}}$} & \multicolumn{2}{|c|}{$E_{1}\left(b_{1}\right)$} & \multicolumn{2}{|c|}{$\emptyset \mathrm{mrad} / \mathrm{h}$} \\
\hline & & & $\begin{array}{c}\mathrm{x}= \\
7.10^{-3} \mathrm{~cm}\end{array}$ & $\begin{array}{c}x= \\
3 \cdot 10^{-1} \mathrm{~cm}\end{array}$ & $\begin{array}{c}\mathrm{x}= \\
7.10^{-3} \mathrm{~cm}\end{array}$ & $\begin{array}{c}\mathrm{x}= \\
3 \cdot 10^{-1} \mathrm{~cm}\end{array}$ \\
\hline 0,15 & $1,5.10^{-3}$ & $3,9 \cdot 10^{-7}$ & 6,3 & 2,6 & $1,3 \cdot 10^{-6}$ & $5.10^{-7}$ \\
\hline 0,3 & $4,56 \cdot 10^{-3}$ & $2,46.10^{-6}$ & 6,5 & 2,8 & $8,5 \cdot 10^{-6}$ & $3,7.10^{-6}$ \\
\hline 0,6 & $3,84 \cdot 10^{-3}$ & $4,20.10^{-6}$ & 6,8 & 3 & $1,43.10^{-5}$ & $6,3 \cdot 10^{-6}$ \\
\hline 1,1 & $2,46 \cdot 10^{-3}$ & $4,55.10^{-6}$ & 7,1 & 3,2 & $1,63.10^{-5}$ & $7,4 \cdot 10^{-6}$ \\
\hline \multirow[t]{2}{*}{1,8} & $2,7 \cdot 10^{-3}$ & $7,30.10^{-6}$ & 7,4 & 3,6 & $2,7 \cdot 10^{-5}$ & $1,33.10^{-5}$ \\
\hline & & & & & $6,8 \cdot 10^{-5}$ & $3,1 \cdot 10^{-5}$ \\
\hline
\end{tabular}

\section{RÉSULTATS}

Débit de dose reçu par la peau : $6,8 \cdot 10^{-5} \mathrm{mrad} / \mathrm{h}$.

Débit de dose reçu par les tissus profonds : $3,1 \cdot 10^{-5} \mathrm{mrad} / \mathrm{h}$. 


\section{ANNEXE B \\ CALCUL DE L'IRRADIATION EXTERNE PAR LE RAYONNEMENT $\beta$}

L'irradiation par le rayonnement $\beta$ peut être importante à faible distance; c'est le cas lorsqu'on est tout près d'une source, ou lorsqu'on se trouve dans une atmosphère contaminée, ou encore si la peau est couverte de poussières radioactives.

On trouvera, ci-dessous, les principaux émetteurs $\beta$ présents dans le minerai d'uranium [2] :

\begin{tabular}{c|c}
\hline \hline $\begin{array}{c}\text { Principaux } \\
\text { émetteurs }\end{array}$ & $\begin{array}{c}\text { Energie maximale } \\
\beta \text { en MeV }\end{array}$ \\
\hline $234 \mathrm{Th}$ & 0,19 \\
$234 \mathrm{~m} \mathrm{~Pa}$ & 2,3 \\
$214 \mathrm{~Pb}$ & 0,65 \\
$214 \mathrm{Bi}$ & 3,2 \\
$210 \mathrm{Bi}$ & 1,17 \\
\hline \hline
\end{tabular}

Seuls figurent, dans ce tableau, les émetteurs dont les particules $\beta$ sont susceptibles d'irradier la couche basale de l'épiderme, c'est-à-dire, celle qui se trouve sous une épaisseur de $7 \mathrm{mg} / \mathrm{cm}^{2}$. Les particules $\beta$ perdent $99 \mathrm{p}$. cent de leur énergie dans la première moitié de leur parcours $R$ [5]; l'irradiation n'est donc significative que si ce parcours est au moins égal à deux fois l'épaisseur-écran à traverser; $R$ doit alors être, dans l'eav, d'au moins $14 \mathrm{mg} / \mathrm{cm}^{2}$, ce qui exige une énergie initiale supérieure à $0,09 \mathrm{MeV}[8, \mathrm{p}$. 70]. Parmi ces particules, seules celles dont l'énergie est supérieure à 1,3 MeV ont un parcours, dans l'eau, d'au moins $600 \mathrm{mg} / \mathrm{cm}^{2}$, et peuvent alors irradier le cristallin et les tissus profonds situés sous $300 \mathrm{mg} / \mathrm{cm}^{2}$.

En un point d'un milieu radioactif suffisamment étendu pour que ses dimensions dans toutes les directions autour du point soient supérieures au parcours maximal des particules, tout se passe comme si le milieu était infini [5]. Il y a équilibre électronique, c'est-à-dire, que l'énergie absorbée dans une certaine masse de la matière constituant le milieu, est égale à l'énergie émise par cette même masse. La dose qui en résulte est donc la dose maximale que l'on puisse trouver dans le milieu source; sa valeur est [5, p. 569] :

$$
I(\mathrm{rad} / \mathrm{h})=2130 C(\mathrm{mCi} / \mathrm{g}) \cdot \bar{E}(\mathrm{MeV}),
$$


en particulier, quand il s'agit d'un radioélément dont les particules $\beta$ ont une énergie initiale $E_{0}$ variant entre zéro et une valeur maximale $E_{m}$ et sont distribuées selon un spectre continu.

$\bar{E}$ est l'énergie moyenne définie par l'énergie totale des particules divisée par leur nombre. La référence [6] donne les valeurs de $\bar{E}$ pour différentes valeurs de $E_{m}$ (de 0,1 à $5 \mathrm{MeV}$ ); on y constate que le rapport $E_{m} / \bar{E}$ varie de 3 à 2,2 .

$C$ est la concentration de l'élément radioactif dans le milieu source.

Si le milieu est limité par une surface plane, la dose en un point de cette surface est, par raison de symétrie, égale à la moitié de la dose précédente :

$$
I_{s}=1065 C \bar{E}(\mathrm{rad} / \mathrm{h}) .
$$

Ceci est vrai si l'épaisseur $h$ du milieu source est supérieure au parcours maximal $R$ des particules $\beta$; si $h$ est inférieur à $R$, le débit de dose à la surface devient :

$$
I_{h}=I_{s}\left(1-e^{-\mu h}\right),
$$

l'atténuation par la matière pouvant être considérée comme étant de forme exponentielle.

$\mu$ est le coefficient d'atténuation apparente dont une expression empirique est donnée ci-après $[5$, p. 570$]$ :

$$
\mu=\frac{18,6}{\left(E_{m}-0,036\right)^{1,37}}\left(\mathrm{~cm}^{2} / \mathrm{g}\right) \text { pour l'eau. }
$$

Ces relations ne peuvent être rigoureuses puisque l'atténuation du faisceau d'électrons dépend évidemment de la forme du spectre.

Le coefficient massique d'atténuation (en $\mathrm{cm}^{2} / \mathrm{g}$ ) se montre peu dépendant de la nature du milieu, c'est-à-dire que le coefficient linéaire d'atténuation $\left(\mathrm{en} \mathrm{cm}^{-1}\right)$ est égal à $\mu \rho ; \mu$ étant le coefficient de l'eau.

L'absorption des particules $\beta$ se poursuit entre la source et le point irradié. Si la distance à parcourir $p$ est inférieure à la moitié du parcours $R$ des particules, l'atténuation est à peu près exponentielle et l'on $\mathrm{a}, \mathrm{au}$ point considéré :

$$
I_{p}=I_{h} e^{-\mu p}(\mathrm{rad} / \mathrm{h}) \text {. }
$$

Ce modèle de calcul peut s'appliquer dans nos trois cas de milieuxsources, étant donné que deux de leurs dimensions, au moins, sont supérieures au parcours $R$. On se trouve ici en présence d'un ensemble de cinq spectres $\beta$; on calculera l'effet de chacun, pris séparément, et l'on fera la somme de ces effets. 


\section{RÉSUltats}

Irradiation $\beta$ par les parois

Il s'agit d'une paroi en minerai d'uranium à $1 \mathrm{p}$. mille défini précédemment. Dans la roche, le parcours $R$ des particules $\beta$ est inférieur à $1 \mathrm{~cm}$. La concentration $C$ de chaque élément radioactif émetteur $\beta$ est de $3,33.10^{-7} \mathrm{mCi} / \mathrm{g}$ de roche.

On a, dans le tableau suivant, les principaux éléments du calcul.

\begin{tabular}{|c|c|c|c|c|c|c|}
\hline \multirow{2}{*}{$\begin{array}{c}\text { Emetteurs } \\
\beta\end{array}$} & \multirow{2}{*}{$\mathrm{E}_{\mathrm{m}}(\mathrm{MeV})$} & \multirow{2}{*}{$\overline{\mathrm{E}}(\mathrm{MeV})$} & \multicolumn{2}{|c|}{ Parcours R $\left(\mathrm{g} / \mathrm{cm}^{2}\right)$} & \multirow{2}{*}{$\underset{\mathrm{Is}}{\mathbf{I}_{\mathbf{s}}}$} & \multirow{2}{*}{$\begin{array}{c}\mu \\
\mathrm{cm}^{2} / \mathrm{g}\end{array}$} \\
\hline & & & air & eau & & \\
\hline $234 \mathrm{Th}$ & 0,19 & 0,07 & $5,3 \cdot 10^{-2}$ & $4,6 \cdot 10^{-2}$ & $2,48.10^{-2}$ & $1,03.10^{3}$ \\
\hline $234 \mathrm{~m} \mathrm{~Pa}$ & 2,3 & 1 & 1,2 & 1,1 & $3,55.10^{-1}$ & 6,08 \\
\hline $214 \mathrm{~Pb}$ & 0,65 & 0,25 & $2,4 \cdot 10^{-1}$ & $2,2.10^{-1}$ & $8,85 \cdot 10^{-2}$ & $1,55.10^{2}$ \\
\hline $214 \mathrm{Bi}$ & 3,26 & 1,5 & 1,7 & 1,5 & $5,33 \cdot 10^{-1}$ & 3,84 \\
\hline $210 \mathrm{Bi}$ & 1,17 & 0,46 & $5,5 \cdot 10^{-1}$ & $4,8 \cdot 10^{-1}$ & $1,63 \cdot 10^{-1}$ & $1,57.10^{1}$ \\
\hline
\end{tabular}

Le point irradié est séparé de la source par une couche d'air et une couche de tissus.

L'épaisseur d'air varie avec la distance; on n'a considéré ici que deux distances, $10 \mathrm{~cm}$ et $1 \mathrm{~m}$. L'épaisseur de tissus à traverser sera de $7 \mathrm{mg} / \mathrm{cm}^{2}$ pour irradier la couche basale de l'épiderme et $300 \mathrm{mg} / \mathrm{cm}^{2}$ pour irradier le cristallin ou les tissus profonds. Dans ces conditions, les valeurs des débits de dose sont les suivantes :

\begin{tabular}{c|c|c}
\hline \hline \multirow{2}{*}{$\begin{array}{c}\text { Distance à } \\
\text { la paroi }(\mathrm{cm})\end{array}$} & \multicolumn{2}{|c}{ Epaisseur des tissus traversés } \\
\cline { 2 - 3 } & $7 \mathrm{mg} / \mathrm{cm}^{2}$ & $300 \mathrm{mg} / \mathrm{cm}^{2}$ \\
\hline 10 & $0,89 \mathrm{mrad} / \mathrm{h}$ & $0,21 \mathrm{mrad} / \mathrm{h}$ \\
100 & $0,26 \mathrm{mrad} / \mathrm{h}$ & $0,12 \mathrm{mrad} / \mathrm{h}$ \\
\hline \hline
\end{tabular}

\section{Irradiation externe $\beta$ par le radon 222}

La concentration $C$ est de $10^{-10} \mathrm{Ci}$ de radon par litre d'air, et le radon est supposé en équilibre radioactif avec ses descendants : ici, plomb 214, bismuth 214 et bismuth 210 . 
Les valeurs calculées du débit de dose sous $7 \mathrm{mg} / \mathrm{cm}^{2}$ et sous $300 \mathrm{mg} / \mathrm{cm}^{2}$ sont les suivantes en fonction de l'épaisseur du milieu-source :

\begin{tabular}{c|c|c}
\hline \hline \multirow{2}{*}{$\begin{array}{c}\text { Epaisseur h } \\
\text { du milieu-source } \\
\text { (air contaminé) } \\
(\mathrm{cm})\end{array}$} & \multicolumn{2}{|c}{$\begin{array}{c}\text { épaisseur } \mathrm{p} \text { des tissus } \\
\text { traversés }\end{array}$} \\
\cline { 2 - 3 } & $7 \mathrm{mg} / \mathrm{cm}^{2}$ & $300 \mathrm{mg} / \mathrm{cm}^{2}$ \\
\hline 100 & $8,4 \cdot 10^{-2} \mathrm{mrad} / \mathrm{h}$ & $1,5 \cdot 10^{-2} \mathrm{mrad} / \mathrm{h}$ \\
209 & $1,2.10^{-2} \mathrm{mrad} / \mathrm{h}$ & $2,5 \cdot 10^{-2} \mathrm{mrad} / \mathrm{h}$ \\
$>1310=\mathrm{R}$ maximal & $1,6.10^{-2} \mathrm{mrad} / \mathrm{h}$ & $3,9.10^{-2} \mathrm{mrad} / \mathrm{h}$ \\
\hline \hline
\end{tabular}

\section{Irradiation $\beta$ par contamination cutanée}

La poussière est supposée identique au minerai d'uranium à $1 \mathrm{p}$. mille décrit précédemment. Elle se dépose uniformément sur la peau et son activité $\alpha$ est de $10^{-6} \mu \mathrm{Ci} / \mathrm{cm}^{2}$, ce qui correspond à une concentration $C$ de $3,33 \cdot 10^{-7}$ $\mathrm{mCi} / \mathrm{g}$ pour chacun des émetteurs et à une épaisseur de poussière égale à $0,6 \mathrm{mg} / \mathrm{cm}^{2}$.

Dans ce cas, les particules $\beta$ sortent d'une source très mince (son épaisseur $h$ est très inférieure au parcours $R$ ) et pénètrent directement dans l'épiderme, sans atténuation par l'air; on obtient ainsi :

$$
\begin{array}{rrr}
6,5 \cdot 10^{-3} \mathrm{mrad} / \mathrm{h} & \text { sous } & 7 \mathrm{mg} / \mathrm{cm}^{2}, \\
6,1 \cdot 10^{-4} \mathrm{mrad} / \mathrm{h} & \text { sous } & 300 \mathrm{mg} / \mathrm{cm}^{2} .
\end{array}
$$

\section{ANNEXE C}

\section{MESURE DU DÉBIT DE DOSE A $1 \mathrm{~m}$ ET A $10 \mathrm{~cm}$ D'UN BLOC DE MINERAI}

On a mesuré l'irradiation due aux rayonnements $\gamma$ et $\beta$, puis au rayonnement $\gamma$ seul émis par un bloc d'uraninite (minerai d'oxydes d'uranium) à très forte teneur et dont le poids était approximativement de $10 \mathrm{~kg}$, la surface frontale de $230 \mathrm{~cm}^{2}$ et la densité de 8 .

Le radiamètre utilisé était une Babyline avec paroi de $300 \mathrm{mg} / \mathrm{cm}^{2} \mathrm{mesu}-$ rant la dose reçue sous la peau ou par le cristallin. Pour mesurer le rayonnement $\gamma$ seul, on a éliminé le rayonnement $\beta$ en interposant une plaque de duralumin épaisse de $6 \mathrm{~mm}$ entre la source et l'appareil.

Étant donné la faible valeur du débit de dose à $1 \mathrm{~m}$, on a opéré en intégrant ce débit de dose sur $1 \mathrm{~h}$. 
Résultats : débit de dose (en $\mathrm{mrad} / \mathrm{h})$

\begin{tabular}{c|c|c}
\hline \hline Débit & à 1 mètre & à 10 cm \\
de dose & (a) \\
\hline$\gamma+\beta$ & 0,68 & 35 \\
$\gamma$ & 0,57 & 27 \\
$\beta$ & 0,11 & 8 \\
& soit 16 p. cent du total & soit 23 p. cent du total \\
\hline \hline
\end{tabular}

(a) La distance est mesurée de la surface du bloc au milieu de la chambre de la Babyline.

\section{BIBLIOGRAPHIE}

[1] Zegers J. C., Pradel J. et Billard F. La radioprotection dans les mines d'uranium françaises. Bull. Inf. A.T.E.N., 1970, $\mathrm{n}^{\circ}$ 81, 5-15.

[2] Lederer M. Table of isotopes (6th ed.), New York, J. Wiley, 1968.

[3] JAEGER R. G. Ed. Engineering compendium on radiation shielding, I, 1968.

[4] Rockwell Th. Reactor shielding design manual, Princeton, Van Nostrand, 1956.

[5] Tubiana M. et coll. Bases physiques de la radiothérapie et de la radiologie, Paris, Masson, 1963.

[6] O'Brien K. Subcutaneous $\beta$-ray dose rate from fission products deposited on the skin. Health Phys., 1974, 26, 122-127.

[7] GrodsteIN G.W. $X$ ray attenuation coefficients from $10 \mathrm{keV}$ to $100 \mathrm{MeV}$. NBS-C-583, 1957.

[8] JOFFRE H. Les problèmes physiques de la radioprotection. In : Génie atomique, Paris, Presses universitaires de France, vol. I, 1963.

[9] Gauzit M. et Kahan Th. Contrôle et protection des réacteurs nucléaires, Paris, Dunod, vol. II, 1957. 